\title{
Unexpected coupling between flow and adsorption in porous media ${ }^{\dagger}$
}

\author{
Jean-Mathieu Vanson, ${ }^{a, b}$ François-Xavier Coudert, ${ }^{c *}$ Benjamin Rotenberg, ${ }^{d}$ Maximilien \\ Levesque ${ }^{a}$ Caroline Tardivat, ${ }^{b}$ Michaela Klotz, ${ }^{b}$ and Anne Boutin ${ }^{a *}$
}

\begin{abstract}
We study the interplay between transport and adsorption in porous systems under a fluid flow, based on a Lattice-Boltzmann scheme extended to account for adsorption. We performed simulations on well-controlled geometries with slit and grooved pores, investigating the influence of adsorption and flow on dispersion coefficient and adsorbed density. In particular, we present a counterintuitive effect where fluid flow induces heterogeneity in the adsorbate, displacing the adsorption equilibrium towards downstream adsorption sites in grooves. We also present an improvement of the adsorption-extended Lattice Boltzmann scheme by introducing the possibility for saturating Langmuir-like adsorption, while earlier work focused on linear adsorption phenomena. We then highlight the impact of this change in situations of high concentration of adsorbate.
\end{abstract}

\section{Introduction}

Transport of molecular fluids ${ }^{1}$ and adsorption of guest species ${ }^{2}$ are among the most studied topics in the domain of nanoporous and macroporous materials. Entire books and countless reviews have been written on these topics, and a large number of theoretical and computational methods have been devised to better understand and predict both phenomena, identifying in particular the influence of key physical and chemical descriptors such as pore geometry, pore connectivity, nature of the internal surface, and strength of host-guest interactions. Such methods range in scale from quantum chemistry calculations and atomistic molecular simulations to the discrete numerical integration methods of computational fluid dynamics.

There are, in the scientific literature, computational studies on the coupling of surface reaction and transport but only a few specifically study the adsorption process and its dependence with transport. ${ }^{3-8}$ Be that as it may, shedding light into this coupling

\footnotetext{
${ }^{a}$ École Normale Supérieure, PSL Research University, Département de Chimie, Sorbonne Universités - UPMC Univ Paris 06, CNRS UMR 8640 PASTEUR, 24 rue Lhomond, 75005 Paris, France. E-mail: anne.boutin@ens.fr

${ }^{b}$ Laboratoire de Synthèse et Fonctionnalisation des Céramiques, UMR 3080 Saint Gobain CREE/CNRS, 550 Avenue Alphonse Jauffret, 84306 Cavaillon, France.

${ }^{c}$ PSL Research University, Chimie ParisTech - CNRS, Institut de Recherche de Chimie Paris, 75005 Paris, France. E-mail: fx.coudert@chimie-paristech.fr

d Sorbonne Universités, UPMC Univ. Paris 06, CNRS, UMR 8234, PHENIX, F-75005 Paris, France.

Email: fx.coudert@chimie-paristech.fr (F.-X. C.); anne.boutin@ens.fr (A. B.)

$\dagger$ Electronic Supplementary Information (ESI) available: Analytical expression for the fraction of adsorbed particles, results on the 3-site analytical model showing the heterogeneity of adsorbed density, description of the relation between reduced units and lattice units in Lattice-Boltzmann simulations.
}

of the two processes is critical in fully understanding both. To give but a single example, even a porous solid with extremely high surface area will perform poorly in adsorption-based separation if the species of interest are not free to efficiently migrate through its pore network.

Recent years have seen the need for computational characterization of the coupling of transport and adsorption, in particular in the context of the renewed interest in hierarchical porous materials for industrial applications. Due to their high modularity, combination of large surface area and pore volume, and enhanced permeability over materials with single pore systems, hierarchically structured porous materials are promising candidates as adsorbent for the separation of gas and liquids, as well as for ion exchange and capture. Potential applications in the liquid phase include water decontamination and pollutant removal, e.g. heavy metals or radioactive ions. ${ }^{9-11}$

The simulation methods classically used to describe adsorption and transport in porous media face hard challenges in dealing with hierarchical pores. On the one hand, at the microscopic scale, adsorption and diffusion are typically studied through atomistic molecular dynamics and Monte Carlo simulations. However, such level of description of both solid and fluid in these approaches has an excessively high computational cost for hierarchical materials where the largest pores may be mesoscopic or macroscopic. On the other hand, larger-scale methods such as computational fluid dynamics can deal with transport in such materials, but do not readily allow for a description of adsorption. In this work, we use the Lattice Boltzmann method, ${ }^{12}$ a mesoscopic lattice-based fluid simulation technique which was recently extended to take into account the adsorption of tracer 
particles at the solid-fluid interface. ${ }^{13-15}$

We first describe the simulation algorithm used to compute our simulations: a Lattice Boltzmann scheme which allows to account for adsorption. We then analyze the effects of adsorption and geometry of the material on transport. We further investigate the influence of transport on adsorption and finally introduce an improvement of the model, by taking into account the saturation of adsorption sites and by examining its consequences.

\section{Methods}

\subsection{The Lattice Boltzmann and moment propagation method}

The Lattice Boltzmann scheme for the numerical simulation of fluid dynamics, which was developed in the late 1980s and has gained much popularity since then, finds its roots in the Lattice Gas Cellular Automata methods. While keeping the idea of the description of the fluid on the nodes of a lattice, the Lattice Boltzmann approach introduces statistical physics through the use of a Boltzmann equation. ${ }^{16-18}$ Unlike classical Computational Fluid Dynamics, the Lattice Boltzmann method does not solve explicitly the Navier-Stokes equations but rather takes a mesoscopic description of the fluid, from which the Navier-Stokes equation can then be derived. ${ }^{19}$ One advantage of the Lattice Boltzmann scheme is that it is relatively easy to incorporate microscopic interactions within the scheme. Of particular interest in our case, the method is efficient for modeling fluid behavior in heterogeneous media such as porous materials. ${ }^{12}$

At the core of the Lattice Boltzmann method is the propagation of the one-particle velocity distribution function $f(r, c, t)$ corresponding to the probability of a particle to be at node $r$ with velocity $c$ at a given time $t$. Time and space are discretized, and so are consequently the velocities, with different models depending on the finite number of speeds $c_{i}$ considered, such as D3Q15, D3Q19 and D3Q27. ${ }^{12,18}$ In this work we used the threedimensional model D3Q19 that has 19 speeds that correspond to the face centers and edge midpoints of the cubic lattice. This model was shown to be a good compromise between precision and computational cost. ${ }^{20}$

The dynamics of the fluid on the lattice is then governed by the following propagation equation:

$$
\begin{aligned}
& f_{i}\left(r+c_{i} \Delta t, t+\Delta t\right) \\
& =f_{i}(r, t)+\frac{\left(f_{i}^{e}(r, t)-f_{i}(r, t)\right)}{\tau}+F_{i}^{e x t},
\end{aligned}
$$

where for simplicity's sake we denote by $f_{i}$ the component of $f$ on speed $i$, i.e. $f_{i}(r, t)=f\left(r, c_{i}, t\right) . \quad f_{i}^{e}$ corresponds to the local Maxwell-Boltzmann equilibrium distribution, $\tau$ is the relaxation time and $F_{i}^{\text {ext }}$ accounts for external forces acting on the fluid (such as a pressure gradient) that will induce some flow. This equation is implemented in our simulations following the method of Ladd and Verberg, ${ }^{21}$ which allows efficient simulation of the dynamics of fluids in complex porous media.

In addition to the Ladd and Verberg method, we want here to compute the dynamical properties of solutes dispersed in the fluid. This problem of tracer dynamics is efficiently ad- dressed by the moment propagation method proposed by Lowe and Frenkel ${ }^{22,23}$ and further validated by Merks et al. ${ }^{24}$ In this method, a propagated quantity $P(r, t)$ is defined on the lattice which evolves in time steps $\Delta t$ as:

$$
\begin{aligned}
P(r, t+\Delta t)= & \sum_{i} P\left(r-c_{i} \Delta t, t\right) p_{i}\left(r-c_{i} \Delta t, t\right) \\
& +P(r, t)\left(1-\sum_{i} p_{i}(r, t)\right),
\end{aligned}
$$

where $p_{i}(r, t)$ represents the probability of leaving node $r$ with speed $c_{i}$ :

$$
p_{i}(r, t)=\frac{f_{i}(r, t)}{\rho(r, t)}-w_{i}+w_{i} \lambda \quad \text { with } \quad \lambda=\frac{2 D_{b}}{v_{T}^{2} \Delta t} .
$$

Here $\rho$ is the fluid density, $w_{i}$ are constant weights depending upon the underlying LB lattice, $D_{b}$ is the diffusion coefficient of the tracers, and $v_{T}$ is the fluid's speed of sound $\left(v_{T}^{2}=\frac{1}{3} \Delta x^{2} / \Delta t^{2}\right.$ for D3Q19, with $\Delta x$ the lattice spacing). For a particular choice of the propagated quantity, namely the probability to arrive at position $r$ at time $t$, weighted by the initial velocity of the tracer (in practice, one quantity is propagated for each component of the velocity), the velocity autocorrelation function $Z$ can be obtained:

$$
Z(t)=\sum_{r} P(r, t)\left(\sum_{i} p_{i}(r, t) c_{i}\right)
$$

Finally, an important quantity in the dynamical properties of the tracers is the dispersion coefficient $K$ to analyze and understand the behavior of particles carried by a fluid. ${ }^{25-27}$ It quantifies the spreading of particles inside the fluid and is defined from the standard deviation of the position of particles as:

$$
K=\lim _{t \rightarrow \infty} \frac{\sigma^{2}}{2 t} \quad \text { where } \quad \sigma^{2}=\langle r-\bar{r}\rangle^{2},
$$

with $\bar{r}$ the average position of the particles at the considered time. In practice, we compute it from the offsetted integration of the velocity auto-correlation function:

$$
K=\int_{0}^{\infty}[Z(t)-Z(\infty)] d t
$$

\subsection{Accounting for adsorption}

There are relatively few studies in the literature accounting for physical adsorption in porous media within the Lattice Boltzmann framework. Argawal et al. developed, in 2005, a Lattice Boltzmann model for one dimentional breakthrough curves simulating the adsorption of toluene on silica gels. ${ }^{28}$ Anderl et al. used Lattice Boltzmann to simulate adsorption and bubble interaction in protein foams, ${ }^{29}$ while Manjhi et al. applied it to the simulation of two-dimensional unsteady state concentration profiles for packed bed adsorbents. ${ }^{30}$ Zalzale et al. used a similar scheme to study the permeability of microstructures of cement pastes, ${ }^{31}$ as did Pham et al. and Tallarek et al. for adsorption on packed beds. ${ }^{7,32}$

In the present work, we use a novel Lattice Boltzmann scheme 
coupling adsorption and tracer dynamics, presented recently by some of the present authors. ${ }^{13-15}$ In this approach, adsorption occurs on interfacial fluid sites, defined as fluid nodes having at least one solid neighboring node accessible through one of the velocities of the D3Q19 model. We consider here the case where adsorbed species do not diffuse on the surface. Adsorption on interfacial site is described as an equilibrium between two populations of solute on the same lattice node: adsorbed and nonadsorbed (free) species. The local adsorption equilibrium between these is dictated by the adsorption and desorption kinetic constants, $k_{a}$ and $k_{d}$, which are the probabilities to adsorb and desorb per surface unit, respectively. The interplay between adsorption and transport is tracked by considering an additional propagated quantity for the adsorbed solute, $P_{\text {ads }}$, and coupling its propagation to that of the free solute:

$$
\begin{aligned}
P_{\mathrm{ads}}(r, t+\Delta t) & =P(r, t) p_{a}+P_{\mathrm{ads}}(r, t)\left(1-p_{d}\right) \\
P(r, t+\Delta t) & =P(r, t)\left(1-p_{a}\right)+P_{\mathrm{ads}}(r, t) p_{d},
\end{aligned}
$$

where $p_{a}=k_{a} \Delta t / \Delta x$ and $p_{d}=k_{d} \Delta t$. At $t=0$, the propagated quantity is initialized from the steady-state densities of adsorbed and free solute obtained by a prior calculation (see details in Section 1.3). Within these approximations, the fraction of adsorbed particles $F_{a}$ in the steady state can be calculated analytically as a function of the number of fluid lattice nodes $N_{f}$, the number of adsorption sites (interfacial nodes) $N_{\text {ads }}$, and the adsorption and desorption coefficients (see Supplementary Information):

$$
F_{a}=\left(1+\frac{p_{d} N_{f}}{p_{a} N_{\mathrm{ads}}}\right)^{-1} .
$$

In addition to the overall fraction of adsorbed species, it is also of interest to know their spatial distribution, which needs not be homogeneous, as shown in Section 3.2. We have thus introduced the calculation of the adsorbed density $D_{\text {ads }}(r, t)$ and free density $D_{\text {free }}(r, t)$ of tracers. Both quantities evolve according to the same rules as the one involved in the computation of $P$ and $P_{\text {ads }}$. In particular, at interfacial nodes they obey:

$$
\begin{aligned}
& D_{\text {ads }}(r, t+\Delta t)=D_{\text {free }}(r, t) p_{a}+D_{\text {ads }}(r, t)\left(1-p_{d}\right) \\
& D_{\text {free }}(r, t+\Delta t)=D_{\text {free }}(r, t)\left(1-p_{a}\right)+D_{\text {ads }}(r, t) p_{d} .
\end{aligned}
$$

At $t=0, D_{\text {free }}$ is set homogeneously in the fluid to $1 / N_{f}$ while $D_{\text {ads }}$ is set to zero.

\subsection{Practical details}

The simulations reported herein are performed with the code laboetie.* Each simulation is composed of three subsequent integration steps. The first one is the flux equilibration, where the velocity and density fields of the fluid itself are converged. This allows computing the transition probabilities for tracer propagation (Eq. 3). The second is the tracer density equilibration, where the free and adsorbed density fields of the tracers are equi- librated following Eq. 9, starting from homogeneous distribution of the tracers in the fluid. The third step is the moment propagation, which describes the motion of particles carried by the fluid and accumulates dynamical information. Throughout this last step, the propagated quantities for free and adsorbed particles are computed, as well as the velocity auto-correlation function, the diffusion coefficient and the dispersion coefficient.

This three-stage simulation procedure is different from the twostage procedure followed by earlier work, ${ }^{13}$ which considered only flux equilibration and moment propagation, and where the adsorbed density was assumed to be homogeneous and at thermodynamic equilibrium. We will show here (Section 2.4 ) that in the presence of fluid flow, the adsorbed density is not identical on all adsorption sites, as solute transport by the fluid induces an heterogeneity. Thus, an extra stage in the simulation procedure is required to equilibrate tracer densities in order to initialize the propagated quantities in Equation 7. Otherwise, if the initial values of the propagated quantities are not representative of the steady state, dynamical information calculated from the Lattice Boltzmann integration will be incorrect.

We used convergence criteria of $10^{-14}$ (in relative step-to-step variation) for the average velocity of the fluid along each direction of space, $10^{-12} \Delta x / \Delta t$ on the value of the velocity autocorrelation function, and $10^{-9}$ for the step-to-step variation of the dispersion coefficient. The tracer diffusion coefficient was fixed throughout all the simulations reported here, at $D_{b}=10^{-2} \Delta x^{2} / \Delta t$. Three-dimensional periodic boundary conditions are enforced. In section 2, results are presented in Lattice-Boltzmann units (reduced units) whereas the real units (S.I units) are employed in section 3. The way to switch between reduced and real units is explained in supplementary information.

\section{Reciprocal influence of transport and ad- sorption}

\subsection{Slit pore geometry}

The first pore geometry studied here, albeit briefly, is that of the slit pore made from two parallel walls. It is very simple yet serves as a good reference for comparison of later results and discussion. The pore width in these simulations was taken to $H=100$ lattice units. In order to study the effect of fluid flow and its coupling to adsorption, we varied both the adsorption equilibrium (by keeping $k_{a}=10^{-1} \Delta x / \Delta t$ constant, and varying $k_{d}$ from $10^{-4}$ to $10^{-2} \Delta t^{-1}$ ) and the external force $F_{\text {ext }}$ (from $4.10^{-6}$ to $2.10^{-5} \Delta p / \Delta x$, with $\Delta p$ the lattice pressure unit, describe in supplementary information). The Figure 1 presents the evolution of the dispersion coefficient, $K$, as a function of the average velocity of the fluid and fraction of adsorbed particles $F_{a}$. Figure 1a has been already shown in ${ }^{13 \dagger}$. These results are consistent with the analytical expression available in the specific case of the slit 

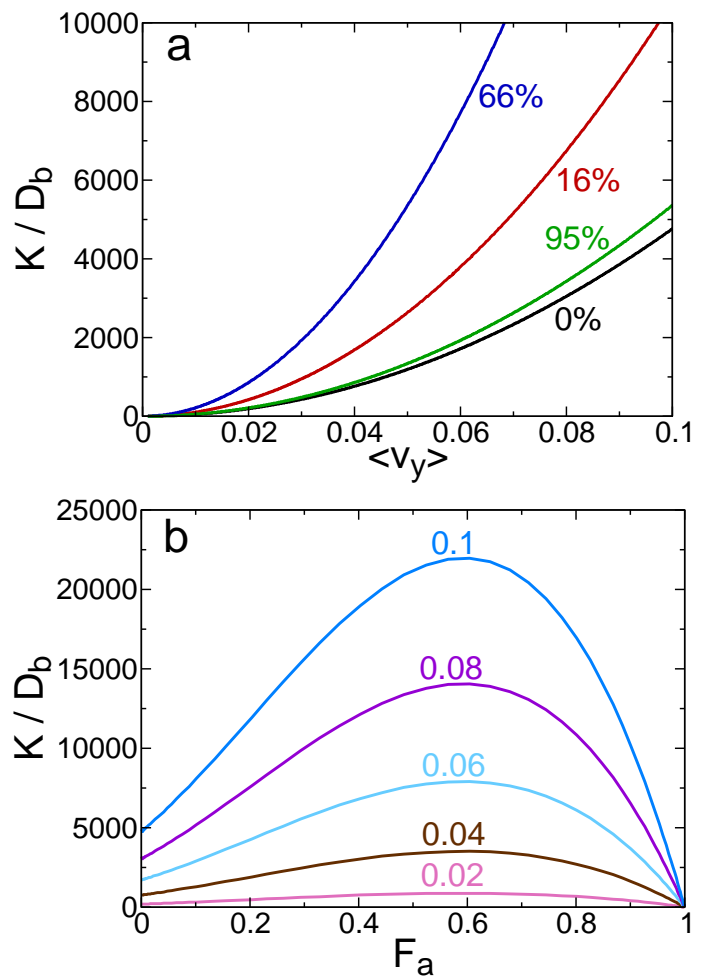

Fig. 1 Adsorption/transport coupling in the slit pore geometry. (a) Influence of the average velocity of the fluid $\left(\left\langle v_{y}\right\rangle\right)$ on the dispersion coefficient $(K)$ normalized by the bulk diffusion coefficient $D_{b}$ for $0 \%$, $16 \%, 66 \%$ and $95 \%$ of particles adsorbed. (b) Influence of the adsorbed fraction $\left(F_{a}\right)$ for some different average velocities of the fluid $(0.02,0.04$, $0.06,0.08,0.1 \Delta x / \Delta t)$.

pore. ${ }^{13 \ddagger}$ :

$$
\begin{aligned}
\frac{K}{D_{b}} & =1-F_{a} \\
& +\operatorname{Pe}^{2}\left(\frac{102 \alpha^{2}+18 \alpha+1}{210(1+2 \alpha)^{3}}+\frac{D_{b}}{H^{2} k_{d}} \frac{2 \alpha}{(1+2 \alpha)^{3}}\right),
\end{aligned}
$$

where $\mathrm{Pe}=H\langle v\rangle / D_{b}$ is the Péclet number and $\alpha=k_{a} / k_{d} H$. We see two effects. First, for a given adsorption strength, that is for a given fraction of adsorbed species, dispersion increases with the fluid average velocity (i.e. Péclet number): Advection spreads out the particles. For low adsorption strengths, the particles stuck to the walls increase dispersion: They are immobile, while the other particles continue to be led away. For high adsorption strengths, the quantity of particles that are led away becomes small since the majority is adsorbed on the wall. Dispersion is maximum for intermediate regimes identified around $F_{a} \approx 0.6$. This maximum can also be seen on earlier work on adsorption in slit pores of Levesque et al. ${ }^{14}$

$\$$ Note there is a term missing in ${ }^{13}$. Re-calculating it from ${ }^{14}$ gives Eq. 10 of the present paper.

\subsection{Crenelated pore}

As a first move toward more complex geometries, we introduce here a corrugated version of the two-dimensional slit-shaped pore. This crenelated pore geometry, depicted in Figure 2, consists in a slit pore with parallelepiped cavities on walls. It is a model of geometries observed experimentally for example in freeze-cast of porous ceramics, ${ }^{33}$ simple enough to allows us to perform and understand the basics of coupling of adsorption and transport in porous materials with complex pore systems. The model is defined by the following geometric characteristics: $L$ is the depthto-depth of the crenelated walls; $\ell$ is the peak-to-peak pore width; $w$ is the width of the crenels; and $h=(L-\ell) / 2$ is their depth. We define $r=h / w$ as the ratio between the height and the width of the crenels, and set $\ell$ constant $(\ell=20)$ in order to maintain constant the effective pore aperture. The system is periodic in $x, y$ and $z$ directions.

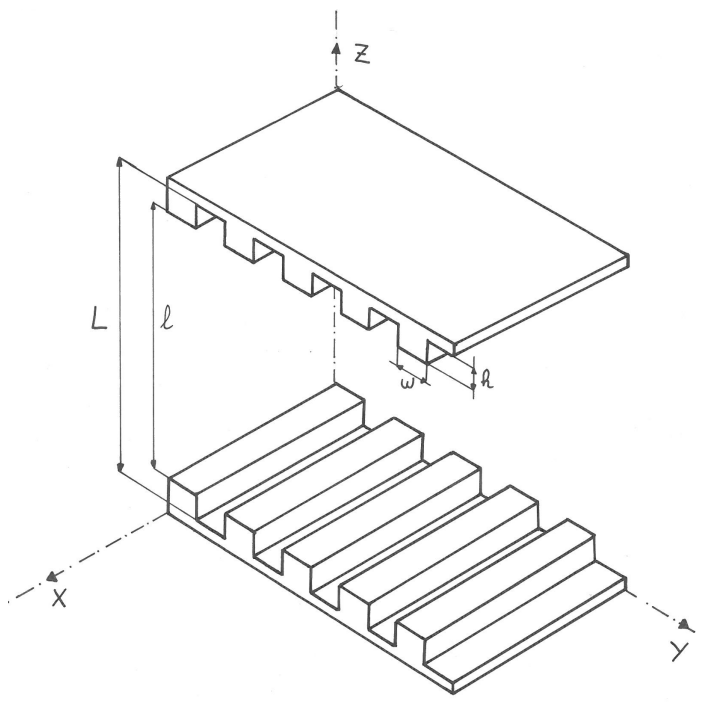

Fig. 2 Geometry of a slit pore having crenels on its walls in isometric view. See text for the geometric characteristics.

In our simulations, we consider an external force (and thus a fluid flow) perpendicular to the crenels, in the $y$ direction. Figure 3 shows the streamlines in this geometry, for a crenel aspect ratio of $r=0.5(h=5 \Delta x, w=10 \Delta x)$ and $F_{\text {ext }}=2.7 \cdot 10^{-4} \Delta p . \Delta x^{-1}$. It also presents the velocity profiles for $y=1$ and $y=10$. We see that the fluid flow is able to enter inside the crenels, though there is a non negligible part of the crenel where the velocity of the fluid is close to zero, indicating the presence of a "dead volume". The flux profile at $y=1$ is a no-slip Poiseuille velocity profile. The velocity profile at $y=10$ is Poiseuille, with additional shoulders due to the pore widening and lower maximun velocity at the center of the pore. The averaged (over $\mathrm{Y}$ ) velocity profile, in this type of geometry, is equivalent to a partial slip profile and the slip length may be quatified analitically. ${ }^{34}$

\subsection{Influence of adsorption and geometry on transport}

We first set out to study the influence of adsorption and geometry on transport, namely the influence of the fraction of adsorbed particles $F_{a}$ (which quantifies the adsorption "strength"), 

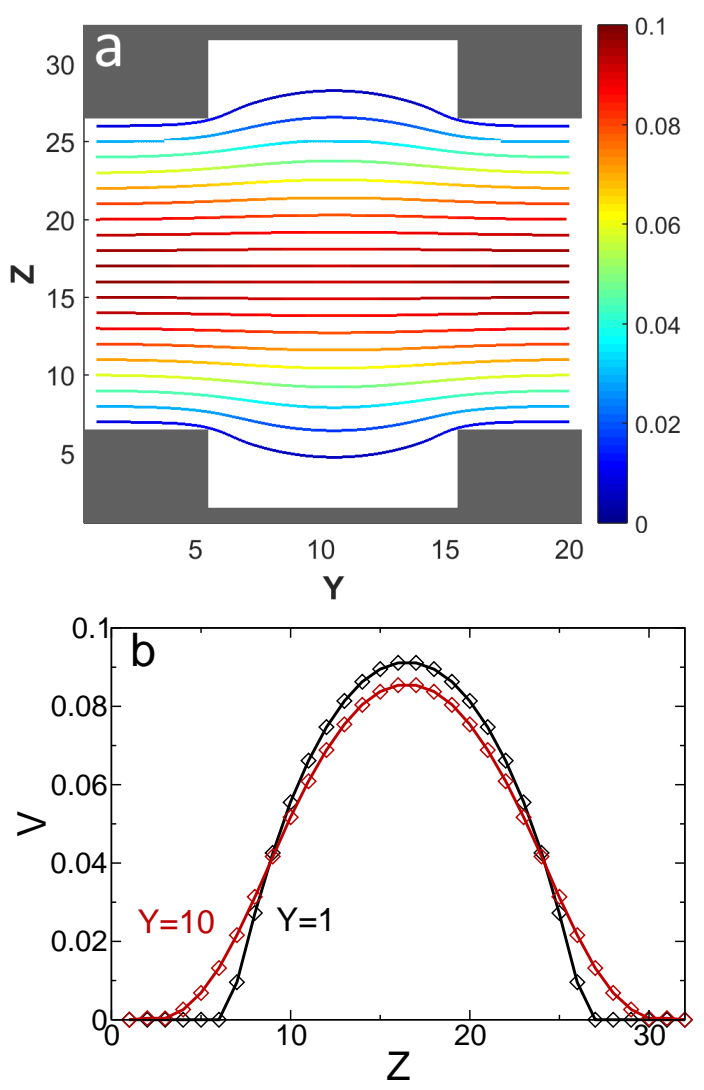

Fig. 3 (a) Streamlines of the flux inside a crenel geometry. Grey areas represent the solid part of the material. The pressure drop is applied along the $y$ axis. The colorbar represents the norm of the velocity (units: $\Delta x / \Delta t$ ) (b) Velocity profiles inside and outside the crenel at $y=1$ and $y=10 . \mathrm{V}$ is the norm of the velocity (units: $\Delta x / \Delta t$ ).

the height-to-width ratio $r$ of the crenels, and the mean fluid velocity on the dispersion of the solute. we take, $l=100 \Delta x$, $r=[0,0.5,1,2,4,10]$ corresponding to $h=[0,5,5,10,20,50]$ nodes and $w=[10,10,5,5,5,5]$ nodes. Figure 4 presents the results of systematic variations of these two parameters. We first see that, as in the case of the slit pore geometry a higher velocity of fluid leads to a larger dispersion (Fig. 4a) and the dispersion varies non-monotonically as a function of $F_{a}$. The value of adsorbed fraction at which dispersion is maximal can be seen to depend on the aspect ratio of the crenelated pore Fig. 4b), indicating the influence of geometry on the transport properties of the solute. Finally, we also see that for $r<1$ the effect of crenel aspect ratio has almost no impact on the dispersion, while for $r>1$ (deep narrow crenels) the dispersion is very sensitive to $r$ and increases alongside it. This strong dispersion is due to the existence of a population of free tracer particles "trapped" inside the narrow crenels, where the velocity of the fluid is almost zero.

Figure $4 c$ represents the evolution of dispersion $\left(K / D_{b}\right)$ as a function of $F_{a}$ in the case $F_{\text {ext }}=0$, i.e. without fluid motion. The tracer particles are thus following a purely diffusive regime, and it can be seen that in this case the dispersion decreases linearly with $F_{a}$. Note that the dispersion in the absence of flow (Fig. 4c) is orders of magnitude smaller than in the presence of flow (Fig. 4a
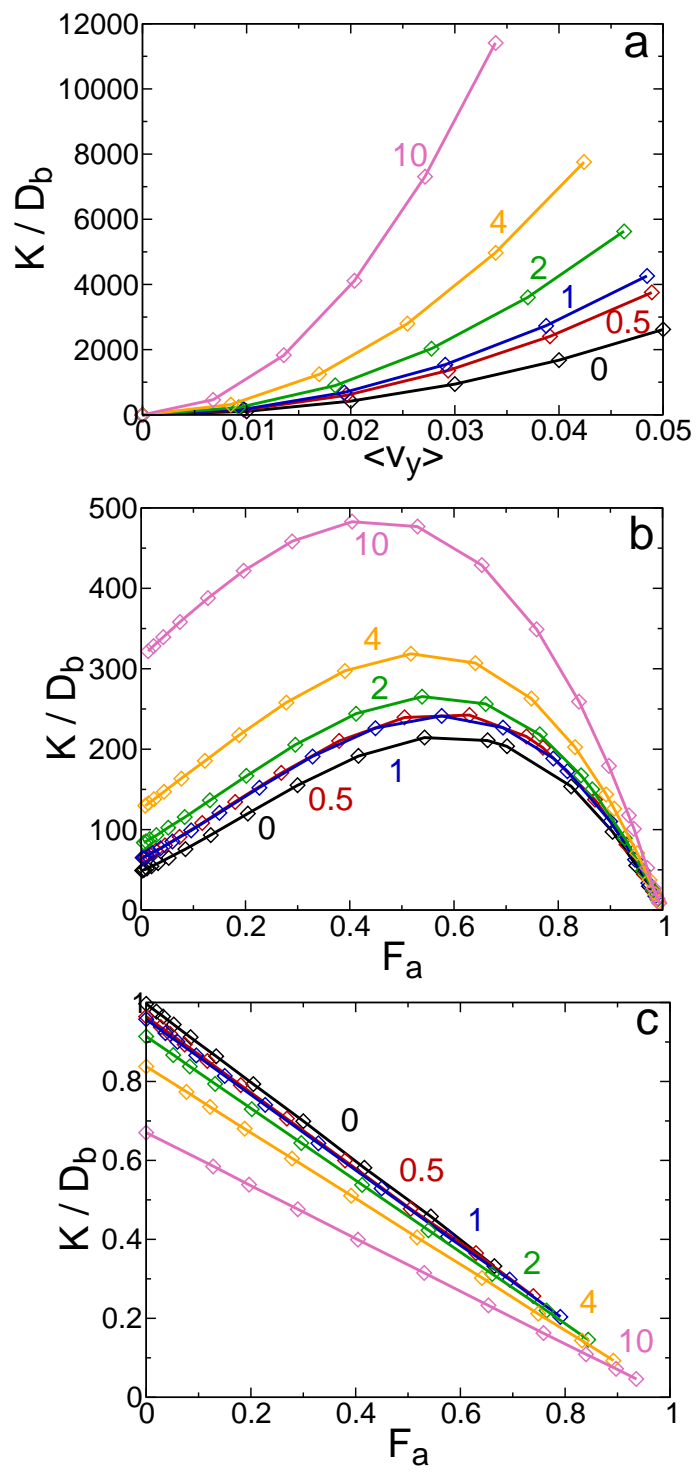

Fig. 4 (a) Influence of the ratio $r=\frac{h}{w}$ and the average velocity of the fluid along y axis $\left(\left\langle v_{y}\right\rangle\right)$ on the dispersion coefficient in the presence of adsorption $\left(k_{a}=0.1 \Delta x / \Delta t\right.$ and $\left.k_{d}=0.01 \Delta t^{-1}\right)$. (b) Influence of the adsorbed fraction $\left(F_{a}\right)$ and the ratio $r=\frac{h}{w}$ on the dispersion coefficient in presence of fluid advection $\left(F_{e x t}=2 \cdot 10^{-6} \Delta p / \Delta x\right)$. (c) Influence of the adsorbed fraction $\left(F_{a}\right)$ and the ratio $r=\frac{h}{w}$ on the dispersion coefficient whithout fluid advection $\left(F_{\text {ext }}=0 \Delta p / \Delta x\right)$

and 4b). Qualitative changes are observed between the vanishing and large $F_{\text {ext }}$ cases, such as the linear to non-motonic variations with $F_{a}$ or the reverse effect of the crenel aspect ratio $r$. Nevertheless, the transition between the two regimes (for $F_{\text {ext }} \rightarrow 0$ ) is continuous. When $F_{\text {ext }} \gg 0$, dispersion is mostly impacted by the advection of fluid: Deeper and narrower crenels retain more particles and dispersion increases consequently. When $F_{\text {ext }}=0$ the dispersion is governed only by diffusion: Deeper and narrower crenels create obstacles to the diffusion, the effective diffusion coefficient (i.e. the dispersion coefficient in theses conditions) decreases. For $F_{\text {ext }}=0$ and $F_{a}=0$ in a slit pore, $K$ is equal to the bulk diffusion coefficient $D_{b}$, as expected. But for crenelated pores the dispersion coefficient is lower than the bulk diffusion coefficient, 
highlighting the impact of the pore geometry on diffusion. If we define $R_{m}=V_{\text {dead }} / V_{\text {fluid }}=h /(L-h)$ as the ratio between the dead volume of fluid, i.e. the volume of fluid inside the crenels and the total volume of fluid, we can see that $\left(1-R_{m}\right)$ and $K / D_{b}$ are similar for all values of $r$ (see Table 1). This, in turn, means that the decrease in the dispersion coefficient is directly related to the dead volume of the crenels.

\begin{tabular}{ccc}
\hline$r$ & $K / D_{b}$ & $1-R_{m}$ \\
\hline 0.5 & 0.96 & 0.95 \\
1 & 0.96 & 0.95 \\
2 & 0.91 & 0.91 \\
4 & 0.84 & 0.83 \\
10 & 0.67 & 0.67 \\
\hline
\end{tabular}

Table 1 Values of the dispersion coefficient $\mathrm{K}$ normalized by the Bulk diffusion coefficient compared with the ratio of dead volume without advection of fluid. The values of $K / D_{b}$ correspond to the diffusive regime without sorption $\left(F_{e x t}=0\right.$ and $\left.F_{a}=0\right)$

\subsection{Influence of transport on adsorption}

We now turn our attention to the reciprocal effect: the influence of transport on adsorption. In order to do so we look at the spatial distribution of adsorbed tracer, characterized by the adsorbed density $D_{\text {ads }}(r)$, for different values of $F_{\text {ext }}$ (and thus average fluid velocity). These simulations were performed at fixed geometry $(h=5, w=10)$ and adsorption strength $\left(k_{a}=10^{-1} \Delta x / \Delta t\right.$, $k_{d}=10^{-3} \Delta t^{-1}$ ). Figure 5 represents the disparities $\phi_{\text {free }}$ and $\phi_{\text {ads }}$ of the free and adsorbed densities:

$$
\phi_{\text {free }}=\frac{D_{\text {free }}-\left\langle D_{\text {free }}\right\rangle}{\left\langle D_{\text {free }}\right\rangle} \quad \text { and } \quad \phi_{\text {ads }}=\frac{D_{\text {ads }}-\left\langle\mathrm{D}_{\mathrm{ads}}\right\rangle}{\left\langle\mathrm{D}_{\mathrm{ads}}\right\rangle},
$$

for $F_{\text {ext }}=0 \Delta p / \Delta x, F_{\text {ext }}=5.10^{-5} \Delta p / \Delta x$, and $F_{\text {ext }}=10^{-4} \Delta p / \Delta x$.

Without fluid flow $\left(F_{\text {ext }}=0\right)$, the adsorbed density is homogeneous: at thermodynamic equilibrium, all adsorption sites are equally populated because they are equivalent. However, as fluid flow is introduced, both the adsorbed and free density distributions in the steady state exhibit some spatial variations. Indeed, on the upstream side of the crenels, when particles desorb the fluid flow brings them to the downstream side and increases locally the adsorbed density. There is a local adsorption equilibrium on each site, between adsorbed and free solutes, so that the adsorbed density is directly proportional to the free density on each interfacial node of the lattice. This demonstrates an influence of fluid transport on solute adsorption. The effect increases with fluid velocity. This effect, predicted here, is somewhat counterintuitive and not commonly expected in the adsorption community. We present, in supporting information, a simple three-site model of adsorption in a flow, for which an analytical solution is available.

In order to provide a quantitative analysis of the influence of fluid flow on the spatial distribution of adsorbates, we present in Figure 6 the evolution of the heterogeneity of the adsorbed
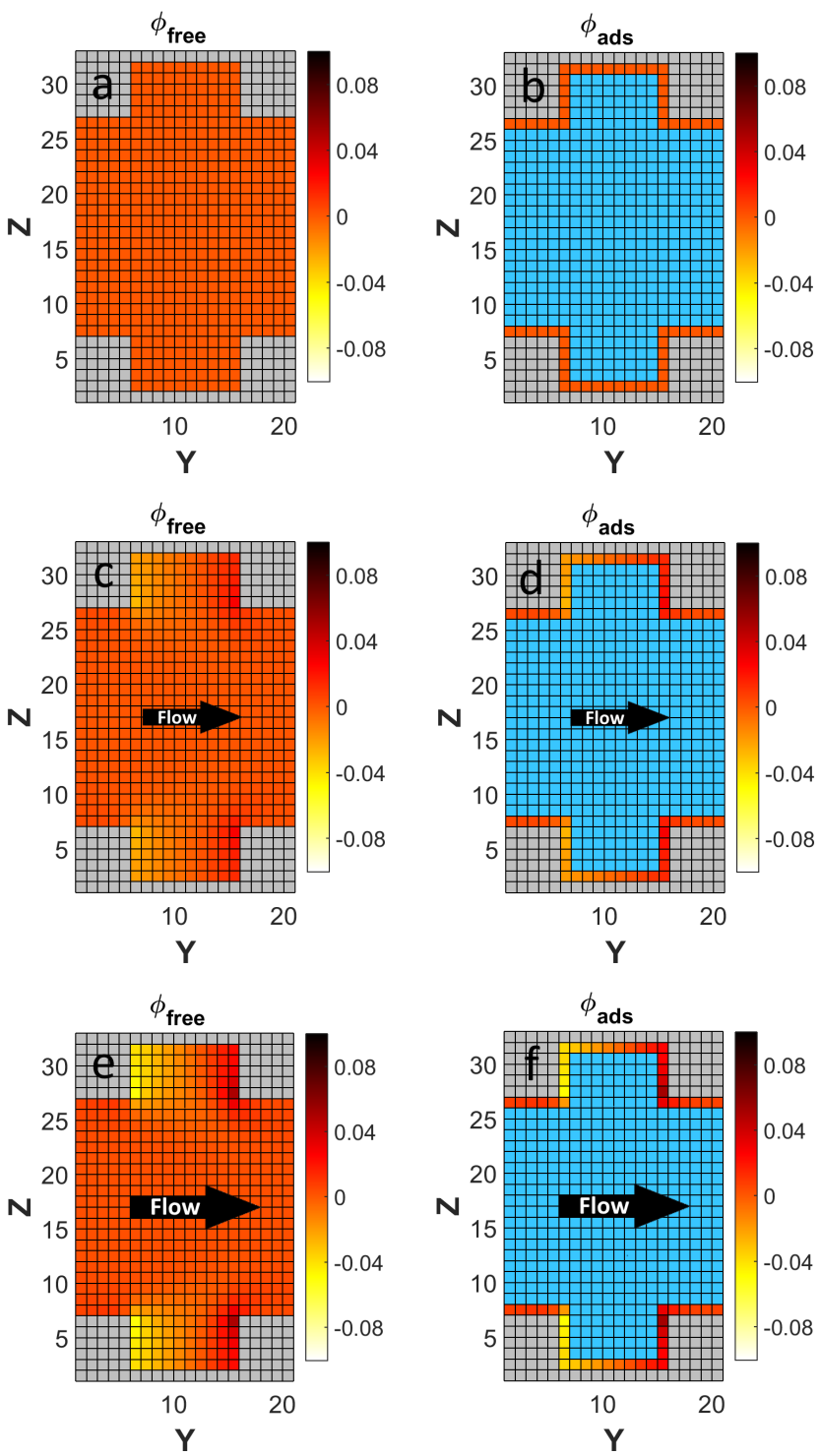

Fig. 5 Disparity of the free and adsorbed densities, $\phi_{\text {free }}$ and $\phi_{\text {ads }}$, due to the flow rate (see Eq. 11). The colored blue zones represent the non-interfacial fluid nodes, where the adsorbed density is equal to zero. Free and adsorbed densities for $F_{e x t}=0$ (a) and (b), $5.10^{-5}$ (c) and (d), and $10^{-4} \Delta p / \Delta x(\mathrm{e})$ and (f).

density as a function of $F_{a}$ and $F_{\text {ext }}$, defined as:

$$
\xi=\sqrt{\left\langle\phi_{\text {ads }}^{2}\right\rangle},
$$

where the average runs over all interfacial nodes. Firstly, we notice that $\xi$ does not depend on $F_{a}$, but increases markedly with $F_{\text {ext }}$ (i.e. the fluid velocity). Secondly, the heterogeneity can reach up to $9 \%$ in our simple model geometry, suggesting that it could have a strong impact on adsorption in more complex and rough geometries. Finally, we note that in our current adsorption model, following a Henry's law with adsorption sites of infinite capacity, this effect of fluid flow on adsorption creates heterogeneity in the adsorbed phase, but does not impact the total amount of adsorbed tracers in the system. The flow favors some adsorption sites compared to others, but does not modify the value of $F_{a}$, which is the 

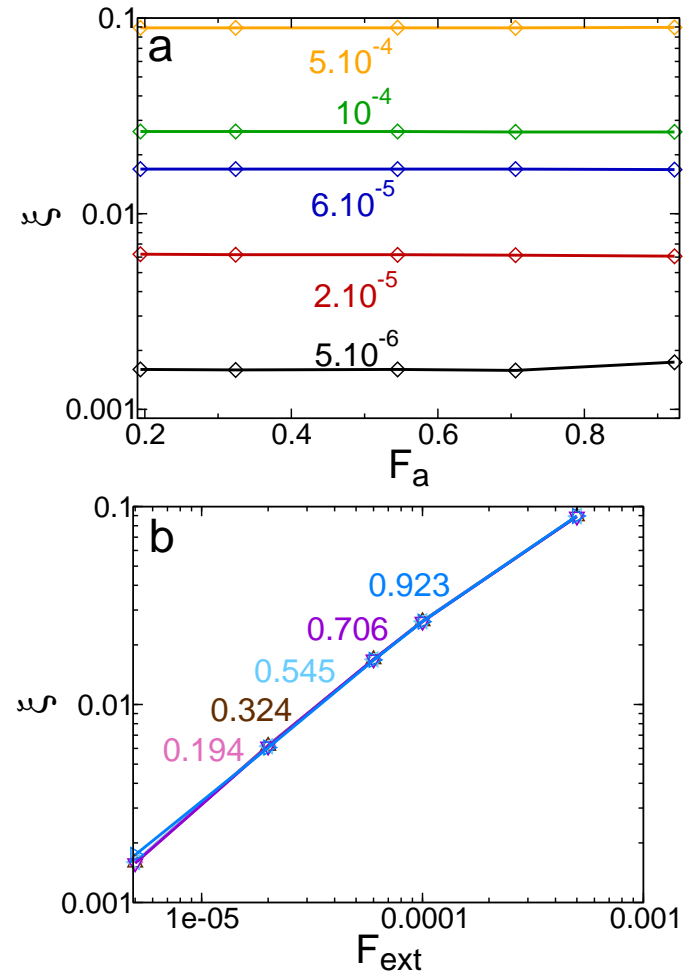

Fig. 6 Adsorbed density disparities (see Eq. 11) in crenelated pores, quantified by the heterogeneity $\xi=\sqrt{\left\langle\phi_{\text {ads }}^{2}\right\rangle}$. (a) Effect of the adsorbed fraction $\left(F_{a}\right)$; colours indicate different external forces $\left(F_{\text {ext }}\right)$. (b) Effect of the external force $\left(F_{\text {ext }}\right)$; colours indicate different adsorbed fractions $\left(F_{a}\right)$.

main observable measured in adsorption experiments.

The observations of Fig. 6 can be rationalized as follows. At steady state, the advection of free tracers by the fluid flow is balanced by a diffusive flux. As a result, a gradient in the free tracer concentration builds up across the groove of width $w$ such that $\left|D_{b} \delta D_{\text {free }} / w\right| \sim\left|D_{\text {free }} v_{y}\right|$. Then, local adsorption equilibrium implies that $\delta D_{\text {ads }} / D_{\text {ads }}=\delta D_{\text {free }} / D_{\text {free }}$. Since the fluid velocity is propotional to the applied force, the heterogeneity thus follows:

$$
\xi=\frac{\lambda F_{\text {ext }}}{D_{b}}
$$

where $\lambda$ is a geometry-dependent parameter independent of $F_{a}$. As $\left\langle v_{y}\right\rangle$ is proportionnal to $F_{\text {ext }}$, we can notice that $\xi \propto \mathrm{Pe}$.

With the current adsorption model the heterogeneity have no influence on $F_{a}$. However, one may anticipate that if the saturation of adsorption sites was taken into account, the total amount of adsorbed tracers may be influenced by the fluid flow and its velocity, because the downstream sites may not accommodate the additional tracers liberated from the upstream ones. This would, in turn, have important consequences for real-life adsorption setups with high concentrations of solutes. In the next section, we therefore extend the above approach to introduce the saturation of adsorption sites.

\section{Model improvement: adsorbent satura- tion}

\subsection{Langmuir adsorption model}

We built upon the existing linear adsorption model (following Henry's law), which considers solute as infinitely diluted tracers, by introducing the saturation of adsorption sites. In order to do so, we use the simplest physical model of saturating adsorbent: the Langmuir adsorption model. In this model, the adsorbed density at each interfacial site is dictated not only by the adsorption and desorption rates, $k_{a}$ and $k_{d}$, but also by the saturation uptake, $D_{\text {max }}$, which is the maximal density that can be adsorbed at any given interfacial lattice node. Similar to the classical statistical physics textbook treatment of the Langmuir model, the propagation equations for the free and adsorbed densities (Equation 9) are modified:

$$
\begin{aligned}
D_{\text {ads }}(r, t+\Delta t)= & {\left[1-\frac{D_{\text {ads }}(r, t)}{D_{\max }}\right] D_{\text {free }}(r, t) p_{a} } \\
& +D_{\text {ads }}(r, t)\left(1-p_{d}\right) \\
D_{\text {free }}(r, t+\Delta t)= & D_{\text {free }}(r, t)\left[1-p_{a}+p_{a} \frac{D_{\text {ads }}(r, t)}{D_{\text {max }}}\right] \\
& +D_{\text {ads }}(r, t) p_{d} .
\end{aligned}
$$

The equations involving the free and adsorbed propagated quantities (Equation 7) are similarly amended:

$$
\begin{aligned}
P_{\mathrm{ads}}(r, t+\Delta t)= & {\left[1-\frac{D_{\mathrm{ads}}(r, t)}{D_{\mathrm{max}}}\right] P(r, t) p_{a} } \\
& +P_{\mathrm{ads}}(r, t)\left(1-p_{d}\right) \\
P(r, t+\Delta t)= & P(r, t)\left[1-p_{a}+p_{a} \frac{D_{\mathrm{ads}}(r, t)}{D_{\max }}\right] \\
& +P_{\mathrm{ads}}(r, t) p_{d} .
\end{aligned}
$$

These modifications were tested on the slit pore and crenelated pore geometries by calculating the total adsorbed quantity as a function of external (free) solute concentration. ${ }^{\S}$ Figure 7 displays the adsorption isotherms thus obtained: For all geometries, the isotherms have the same shape and fit perfectly with a Langmuir equation:

$$
n_{\mathrm{ads}}\left(C_{\mathrm{ext}}\right)=\frac{Q_{\mathrm{max}}}{m_{s}} \frac{\kappa C_{\mathrm{ext}}}{1+\kappa C_{\mathrm{ext}}},
$$

with $C_{\text {ext }}=C_{\text {tot }}\left(1-F_{a}\right)$ the concentration of free tracers, $Q_{\max }=$ $D_{\max } N_{\text {ads }}$ the adsorption capacity of the material and $\kappa=$ $k_{a} / k_{d} D_{\max }$ and $m_{s}=V_{s} \rho_{s}$ the mass of the solid ( $V_{s}$ is the volume of the solid and $\rho_{s}$ is the volumetric mass of the solid. In the following we introduce in the fluid an initial solute concentation

$\S$ We perform simulations in a crenelated pore geometry with $\ell=100, h=5, w=10$, $F_{\text {ext }}=3.65 .10^{13} \mathrm{~Pa} . \mathrm{m}^{-1}, D_{b}=6.042 .10^{-8} \mathrm{~m}^{2} . \mathrm{s}^{-1}, k_{a}=604.2 \mathrm{~m} . \mathrm{s}^{-1}, k_{d}=6.04 .10^{9} \mathrm{~s}^{-1}$, $P_{\max }=1.88 \cdot 10^{-7} \mathrm{~kg} \cdot \mathrm{m}^{-2}, \Delta x=10^{-9} \mathrm{~m}, v_{\text {fluid }}=1.007 \cdot 10^{-6} \mathrm{~m}^{2} / \mathrm{s}, \rho_{\text {fluid }}=1000 \mathrm{~kg} \cdot \mathrm{m}^{-3}$, $\rho_{\text {solid }}=3970 \mathrm{~kg} \cdot \mathrm{m}^{-3}$ 
$C_{t o t}=Q_{\max } S_{s} / V_{p}$ (with $S_{s}$ the specific surface area and $V_{p}$ the pore volume) that corresponds exactly to the amount of solute that can be adsorbed on the surface.

In particular, increasing the roughness, $r$, of the pore surface leads to higher total adsorption capacity $Q_{\max }$, due to a higher specific surface area.

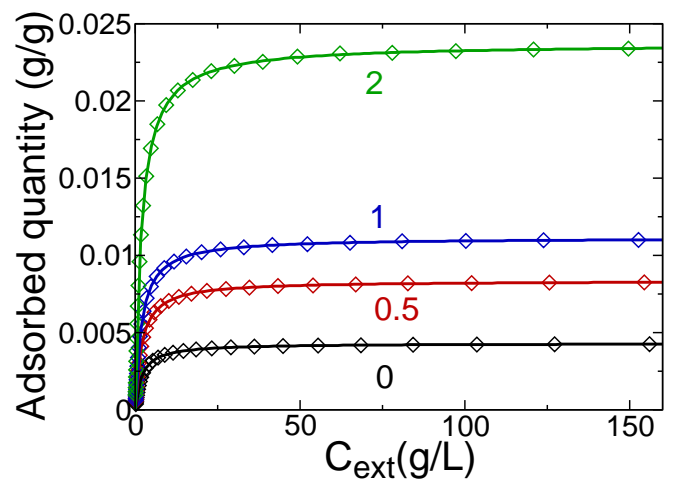

Fig. 7 Adsorption isotherm for different crenelated pore geometries with $r=0$ (slit pore, black), $r=0.5$ (red), $r=1$ (blue) and $r=2$ (green). Diamonds represent numerical data and straight lines correspond to their fit with Eq. 16.

\subsection{Influence on adsorbed density}

With the model of saturating adsorption described above, we now turn our attention to the impact of the saturation uptake and solute concentration on the adsorbed density. We investigated this on a crenelated pore geometry with $h=5$ and $w=10(r=0.5)$. Figure 8 presents the influence of the adsorption strength $\left(k_{a} / k_{d}\right)$ and fluid flow (which is directly linked to the external force $F_{\text {ext }}$ ) on the adsorbed fraction and the adsorbed density. Fig. 8a and 8b can be compared to simulations on a model without saturation of adsorption sites (Fig. 6). Introducing saturation in the adsorption model significantly changes the behavior of $\xi$ as a function of $F_{a}$. When adsorbent saturation is taken into account, the heterogeneity is no more independent of the adsorption strength, but rather diminishes as $F_{a}$ increases. This is because the adsorption site "favored" by the fluid flow (the downstream part of the crenel) is limited in the quantity of solute it can adsorb, leading to smaller $\xi$.

The Figure 8c shows the influence of fluid flow (through $F_{\text {ext }}$ ) on the adsorbed fraction $\left(F_{a}\right)$. It can be seen that, in the case of a saturating adsorbent, there is an effect of fluid flow on the adsorbed fraction: Increasing the fluid velocity leads to less adsorption, because, unlike previously, the sites favourized by the flow have a saturation level. This effect, however, is quite limited in practice, with an impact of $4 \%$ in the most unfavorable case simulated for an extremely high fluid velocity. Nevertheless, the present simulations are performed on a relatively smooth and regular geometry, and we predict that the impact of fluid flow on adsorption will be stronger in more complex geometries.

Finally, Figure 9 shows the evolution of the heterogeneity $\xi$ of the adsorbed density as a function of the external concentration of solute, for various values of the external force. As seen previously,
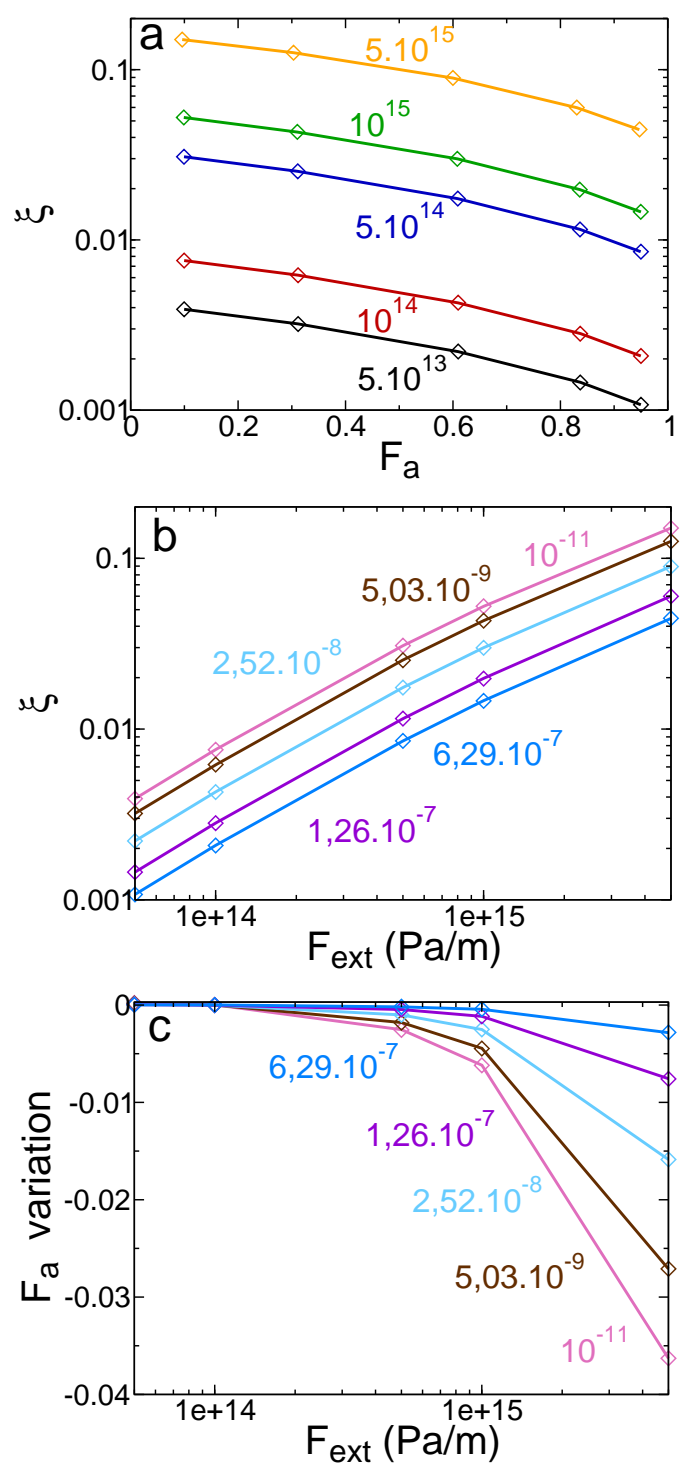

Fig. 8 Adsorbed density heterogeneities due to fluid flow in crenelated pores. (a) Heterogeneity $\xi=\sqrt{\left\langle\phi_{\text {ads }}^{2}\right\rangle}$ as a function of the adsorbed fraction $F_{a}$; colors indicate different values of the external force $F_{e x t}$. (b) Heterogeneity $\xi$ as a function of the external force $F_{\text {ext }}$ applied along the $y$ direction; colors indicate different values of $k_{a} / k_{d}$. (c) Relative deviation of $F_{a}$ compared to its value at $F_{e x t}=0$, as a function of $F_{e x t}$; colors indicate several values of $k_{a} / k_{d} . D_{\max }$ is equal to $10^{-7} \mathrm{Kg} \cdot \mathrm{m}^{-3}$ and the initial concentration of species in the fluid $\left(C_{i}\right)$ is equal to $9.44 \mathrm{~g} \cdot \mathrm{L}^{-1}$.

increases in $F_{\text {ext }}$ lead to an increase of the heterogeneity. In addition, for all values of $F_{\text {ext }}$, the heterogeneity of adsorption shows two regimes as a function of $C_{\text {ext }}$. At low concentration, i.e. in the linear adsorption regime, the heterogeneity is constant. At higher concentration, saturation of the most populated adsorption sites is reached, and the heterogeneity diminishes rapidly. At very high concentration, all adsorption sites are saturated and thus equally populated.

The discussion leading to Eq. 13 also applies to the present case, but the local adsorption equilibrium is modified by the saturation of adsorption sites as: $\delta D_{a d s} / D_{a d s}=\left(\delta D_{\text {free }} / D_{\text {free }}\right) /(1+$ $\kappa C_{\text {ext }}$ ), with $\kappa=k_{a} / k_{d} D_{\max }$ as in Eq. (16). The heterogeneity now 


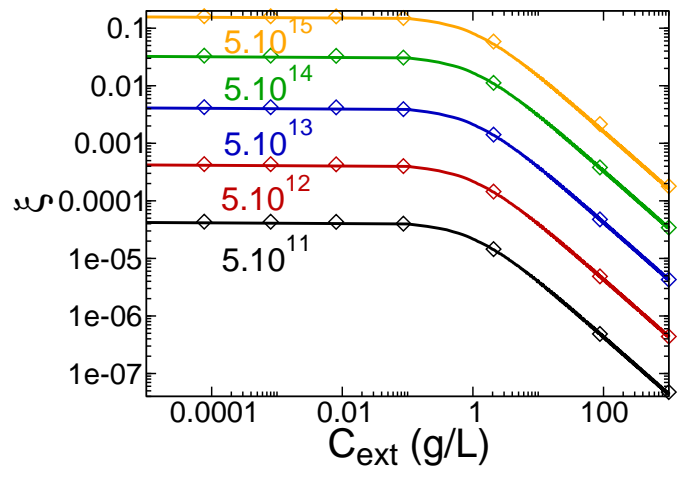

Fig. 9 Adsorbed density heterogeneities due to fluid flow in crenelated pores. Heterogeneity $\xi=\sqrt{\left\langle\phi_{\text {ads }}^{2}\right\rangle}$ as a function of the external concentration $C_{e x t}$. Colors indicate several values of external forces $\left(5.10^{11}, 5.10^{12}, 5.10^{13}, 5.10^{14}, 5.10^{15} \mathrm{~Pa}^{-1}\right) . D_{\max }$ is equal to $10^{-7} \mathrm{~kg} \cdot \mathrm{m}^{-3}$. Symbols indicate simulation data, while straight lines correspond to Eq. 17.

satisfies:

$$
\xi\left(C_{e x t}\right)=\frac{\lambda F_{\text {ext }}}{D_{b}} \frac{1}{1+\kappa C_{\text {ext }}},
$$

in good agreement with the simulation results of Fig. 9.

\section{Conclusion}

In this study we used a recent Lattice Boltzmann model accounting for transport and adsorption. This useful tool allowed us to show and analyze the interdependence between these two coupled phenomena: Not only the effect of tracer adsorption on its transport, but also a counter-intuitive effect of transport on adsorption leading to disparities in the adsorbed density. We then extended the model to account for the saturation of the adsorption sites. This allowed us to explore a wide range of solute concentrations. Taking into account such transport-induced heterogeneity may improve the design of materials targeted to specific applications, e.g. by optimizing the distribution of reactive sites at the surface. The results presented here open the way to the analysis of these effects in more complex geometries, in particular the impact of rugosity on transport and adsorption. As the model is based on a 3D Lattice grid the geometry may be directly applied to real structures, e.g. generated from tomography imaging.

\section{Acknowledgements}

This work was supported by the ANRT through CIFRE sponsorship 1262/2013. We thank Benoit Coasne, Lyderic Bocquet and JeanMichel Drouin for fruitful discussions.

\section{References}

1 M. Sahimi, Flow and Transport in Porous Media and Fractured Rock: From Classical Methods to Modern Approaches, Wiley, 2012.

2 R. M. A. Roque-Malherbe, Adsorption and diffusion in nanoporous materials, Taylor \& Francis, 2007.

3 B. Coasne, A. Galarneau, C. Girardin, F. Fajula and F. Villemot, Langmuir, 2013, 29, 7864-7875.
4 F. Villemot, A. Galarneau and B. Coasne, J. Phys. Chem., 2014, 118, 7423-7433.

5 H. yanagihara, K. Yamashita, A. Endo and H. Daiguji, J. Phys. Chem., 2013, 117, 21795-21802.

6 A. Boţan, F.-J. Ulm, R. J.-M. Pellenq and B. Coasne, Phys. Rev. E, 2015, 91, 032133.

7 D. Hlushkou, F. Gritti, A. Daneyko, G. Guiochon and U. Tallarek, J. Phys. Chem., 2013, 117, 22974-22985.

8 D. Hlushkou, F. Gritti, G. Guiochon, A. Seidel-Morgenstern and U. Tallarek, Anal. Chem., 2014, 86, 4463-4470.

9 M. Hua, S. Zhang, B. Pan, W. Zhang, L. Lv and Q. Zhang, J. Hazard. Mater., 2012, 211-212, 317 - 331.

10 F. Fu and Q. Wang, J. Environ. Manage., 2011, 92, 407 - 418.

11 S. K. R. Yadanaparthi, D. Graybill and R. von Wandruszka, J. Hazard. Mater., 2009, 171, 1 - 15.

12 S. Succi, The Lattice-Boltzmann equation for fluid dynamics and beyond, Oxford Science publications, 2001.

13 M. Levesque, M. Duvail, I. Pagonabarraga, D. Frenkel and B. Rotenberg, Phys. Rev. E, 2013, 88, 013308.

14 M. Levesque, O. Benichou, R. Voituriez and B. Rotenberg, Phys. Rev. E, 2012, 86, 1539-3755.

15 M. Levesque, O. B'enichou and B. Rotenberg, J. Chem. Phys., 2013, 138, -.

16 U. Frisch, D. d'Humi'eres, B. Hasslacher, P. Lallemand, Y. Pomeau and J.-P. Rivet, Complex Systems, 1987, 1, 649707.

17 G. R. McNamara and G. Zanetti, Phys. Rev. Lett., 1988, 61, 2332-2335.

18 Y. H. Qian, D. D’Humi‘eres and P. Lallemand, EPL, 1992, 17, 479.

19 U. Frisch, B. Hasslacher and Y. Pomeau, Phys. Rev. Lett., 1986, 56, 1505-1509.

20 R. Mei, W. Shyy, D. Yu and L.-S. Luo, J. Comput. Phys., 2000, 161, $680-699$.

21 A. J. C. Ladd and R. Verberg, Phys. Rev. E, 2001, 104, 11911251.

22 C. Lowe and D. Frenkel, Physica A, 1995, 220, 251 - 260.

23 C. P. Lowe and D. Frenkel, Phys. Rev. Lett., 1996, 77, 45524555.

24 R. Merks, A. Hoekstra and P. Sloot, J. Comput. Phys., 2002, 183, 563 - 576 .

25 J. M. P. Q. Delgado, Chem. Eng. Res. Des., 2007, 85(A9), $1245-1252$.

26 B. Bijeljic and M. Blunt, Water Resour. Res., 2007, 43, W12S11.

27 S. Whitaker, AIChE Journal, 1967, 13, 420-427.

28 S. Agarwal, N. Verma and D. Mewes, Heat Mass Transfer, 2005, 41, 843-854.

29 D. Anderl, M. Bauer, C. Rauh, U. Rude and A. Delgado, Food Funct., 2014, 5, 755-763.

30 N. Manjhi, N. Verma, K. Salem and D. Mewes, Chem. Eng. Sci., 2006, 61, 2510 - 2521.

31 M. Zalzale and P. McDonald, Cem. Concr. Res., 2012, 42, 1601 
$-1610$.

32 N. H. Pham, D. P. Swatske, J. H. Harwell, B.-J. Shiau and D. V. Papavassiliou, Heat Mass Transfer, 2014, 72, 319 - 328.

33 S. Deville, Advanced Engineering Materials, 2008, 10, 155-
169.

34 C. Ybert, C. Barentin, C. Cottin-Bizonne, P. Joseph and L. Bocquet, Phys. Fluids, 2007, 19, -- 